\title{
An altered microbiome in a Parkinson's disease model Drosophila melanogaster has a negative effect on development
}

Jade Parker-Character

Midwestern University

David R. Hager

Midwestern University

Tanner B. Call

Midwestern University

Zachary S. Pickup

Midwestern University

Scott A. Turnbull

Midwestern University

Evan M. Marshman

Brigham Young University

Shaleen B. Korch

Midwestern University

John M. Chaston

Brigham Young University

Gerald B. Call ( $\square$ gcall@midwestern.edu )

Midwestern University

\section{Research Article}

Keywords: Parkinson's disease (PD), model organism, Drosophila melanogaster, PRKN gene, parkin (park)

Posted Date: September 29th, 2021

DOI: https://doi.org/10.21203/rs.3.rs-909182/v1

License: (1) (i) This work is licensed under a Creative Commons Attribution 4.0 International License.

Read Full License

Version of Record: A version of this preprint was published at Scientific Reports on December 1st, 2021. See the published version at https://doi.org/10.1038/s41598-021-02624-1. 

1 An altered microbiome in a Parkinson's disease model Drosophila melanogaster has a negative effect

2 on development

3

4 Jade Parker-Character ${ }^{1}$, David R. Hager², Tanner B. Call ${ }^{2}$, Zachary S. Pickup ${ }^{1}$, Scott A. Turnbull², Evan M.

5 Marshman ${ }^{3}$, Shaleen B. Korch ${ }^{4}$, John M. Chaston ${ }^{3}$, Gerald B. Call ${ }^{4 *}$

$6 \quad{ }^{1}$ Biomedical Sciences Program, College of Graduate Studies, Midwestern University, Glendale, AZ, USA

$7 \quad{ }^{2}$ Arizona College of Osteopathic Medicine, Midwestern University, Glendale, AZ, USA

$8{ }^{3}$ Department of Plant and Wildlife Sciences, College of Life Sciences, Brigham Young University, Provo,

9 UT, USA

$10{ }^{4}$ Department of Pharmacology, College of Graduate Studies, Midwestern University, Glendale, AZ, USA

$11 *$ Corresponding author, gcall@midwestern.edu 


\section{Abstract}

14 It is well-established that there are differences in the fecal microbiota composition between Parkinson's

15 disease (PD) patients and control populations, but the mechanisms underlying these differences are not

16 yet fully understood. To begin to close the gap between description and mechanism we studied the

17 relationship between the microbiota and PD in a model organism, Drosophila melanogaster. First, fecal

18 transfers were performed with a D. melanogaster model of PD that had a mutation in the parkin (park ${ }^{25}$ )

19 gene. Results indicate that the PD model feces had a negative effect on both pupation and eclosion in

20 both control and park $^{25}$ flies, with a greater effect in PD model flies. Analysis of the microbiota

21 composition revealed differences between the control and park ${ }^{25}$ flies, consistent with many human

22 studies. Conversely, gnotobiotic treatment of axenic embryos with feces-derived bacterial cultures did

23 not affect eclosure. We speculate this result might be due to similarities in bacterial prevalence

24 between mutant and control feces. Further, we confirmed a bacteria-potentiated impact on mutant and

25 control fly phenotypes by measuring eclosure rate in park $^{25}$ flies that were mono-associated with

26 members of the fly microbiota. Both the fecal transfer and the mono-association results indicate a host

27 genotype-microbiota interaction. Overall, this study concludes functional effects of the fly microbiota on

28 PD model flies, providing support to the developing body of knowledge regarding the influence of the 29 microbiota on PD. 


\section{Introduction}

Parkinson's disease (PD) is the second most common neurodegenerative disease affecting more

33 than ten million people worldwide ${ }^{1}$. Most cases of PD are idiopathic, while a small percentage is genetic

34 in origin ${ }^{2}$. Of these genetic cases, mutation of the $P R K N$ gene contributes to approximately $50 \%$ of all

35 autosomal recessive juvenile parkinsonism ${ }^{3}$. Mutation of the Drosophila melanogaster ortholog of the

$36 \quad P R K N$ gene, parkin (park), leads to a tenable model of PD that has many similarities to PD patients:

37 selective loss of dopaminergic neurons, decreased motor function, loss of olfaction, reduced lifespan, mitochondrial dysfunction and others ${ }^{4-7}$. The relative ease of use of flies and powerful genetic tools available in this fly PD model has contributed greatly to the study and understanding of PD ${ }^{8}$.

$4270 \%$ of PD patients ${ }^{9}$. There have been a number studies that have demonstrated that the gut

43 microbiota is altered in PD patients compared to healthy control individuals ${ }^{10}$ and it has been

44 hypothesized that this altered microbiota is largely responsible for many of the GI disorders observed.

45 Beyond this, the altered PD microbiota has been hypothesized to play a role in non-GI PD symptoms,

46 specifically related to the gut-brain axis. In support of this, PD patient fecal transplant into germ-free PD

47 model mice produced an increase in motor deficits compared to PD model mice with a healthy donor

48 fecal transplant ${ }^{11}$. Taken together, the results from these previous studies prompted our investigation

49 of the microbiota in the park mutant fly model.

Relative to humans and other mammals, the $D$. melanogaster microbiota is low-abundance and

51 low-diversity, making it simpler and easier to study microbiota interactions. Laboratory and wild flies are

52 typically colonized by $10^{4}-10^{5}$ microorganisms, and the 2-5 most abundant isolates often represent >

$5390 \%$ of the microbial community ${ }^{12-14}$. The most represented bacteria in the gut are usually acetic acid 
$54(\mathrm{AAB})$ and lactic acid bacteria ( $\mathrm{LAB}$ ), especially members of the genera Acetobacter and Lactobacillus,

55 respectively, and Enterobacteriaceae. Similar to mammals, the fly microbiota composition is determined

56 by both fly genotype and diet ${ }^{14-17}$. Fly larvae possess no gut microbes upon hatching and thus obtain and

57 develop their microbiota from both the environment and food source. Because there is no evidence of

58 high-fidelity host-mediated acquisition or retention of specific microorganism within or across

59 generations, the Drosophila microbiota is 'inconstant'; although some bacterial isolates colonize and

60 persist within the gut better than others ${ }^{18-20}$. A previous analysis of the microbiota in a PD fly model

61 revealed differences in the diversity, but not specific taxonomic changes, between the microbiota of

62 control and PD-model flies ${ }^{21}$. The intracellular endosymbiont Wolbachia is also a common inhabitant of

63 the reproductive tract of Drosophila and, unlike the gut microbiota, is transmitted from mother to

64 offspring within the $\mathrm{egg}^{22}$.

The association between Drosophila and its microbiota is experimentally tractable: bacteria-free embryos are readily derived by bleach treatment and members of the Drosophila microbiota can be

67 isolated in pure culture in the laboratory. Inoculating bacteria-free fly embryos with a defined microbial species or community is called gnotobiotic culture and permits exquisite dissection of the contributions of individual microorganisms to specific fly phenotypes ${ }^{23}$. Adding back one or more bacterial species to the same genotype of sterile fly embryos permits the detection of the magnitude of variation in host

71 traits that is due to the microbiota ${ }^{24}$. Unlike the gut microbiota, bleach treatment does not eliminate

72 Wolbachia from the fly embryos.

In this study we sought to better define the relationship between the microbiota and a $D$.

74 melanogaster PD model by addressing two major questions: 1) Does microbiota manipulation, including

75 via fecal transfer, bacterial-elimination, or gnotobiotic culture affect development, an early and

76 fundamental biologic process,-in a PD-fly model? 2) Does the microbiota vary between control and PD 
77

78

79

80

81

82

83

84

85

86

87

model flies? This study aims to address these questions by measuring fly pupation, eclosion, and/or microbiota composition under a variety of conventional and gnotobiotic culture conditions.

\section{Results}

\section{Fecal transfer from park $^{25}$ flies reduces total pupation rates}

To determine whether differences in the microbiome between control and park ${ }^{25}$ flies might contribute to variation in fly phenotypes we compared the pupation rate of flies that received fecal transfers from control and park ${ }^{25}$ donors. Fecal transfers were performed by allowing males to defecate on cooked but not autoclaved diet vials for 3 days before transferring fly embryos to the feces-seeded diet. The park ${ }^{25}$ feces reduced the total pupation rate of all fly genotypes when compared to the embryos that were placed on food that contained control feces (post-hoc Tukey test: $\mathrm{P}<0.0001$ for all three; Fig. 1). Each of the pupation rates are based off the 60 embryos placed on the food, such that both the park ${ }^{25}$ heterozygous and homozygous pupae numbers come from the same 60 embryos. Twoway ANOVA analysis revealed that $86 \%$ of the variation is due to genotype $(P<0.0001)$, which is expected given the effects of the park ${ }^{25}$ mutation. Additionally, $3.6 \%$ of the variation is due to the fecal transfer effect $(P<0.0001)$. When analyzing daily pupation rates, the homozygous park ${ }^{25}$ flies were the only genotype that had reduced pupation rates on two consecutive days when they received feces from park $^{25}$ mutants, suggesting that the park ${ }^{25}$ homozygous flies are more susceptible to the detrimental effects of the park ${ }^{25}$ fecal transfer (Supplementary Fig. S1). Together, these results identify a negative effect on flies of multiple genotypes when they received a fecal transfer from park ${ }^{25}$ versus control flies.

\section{The park $^{25}$ feces reduces fly eclosion rates}


Eclosion rates for each fly genotype-fecal transfer combination were determined by dividing the

99 number of flies that eclosed by the total number of pupae that had developed for that genotype. Figure

$1002 \mathrm{2A}$ shows that all three fly genotypes experienced a reduced eclosion rate when placed on the park ${ }^{25}$

101 feces compared to the control feces (control and homozygous park ${ }^{25}, \mathrm{P}<0.0001$; heterozygous park ${ }^{25}$, P

$102=0.0101)$. As with pupation, the majority of variation identified was due to fly genotype $(54 \%, \mathrm{P}<$

1030.0001 ), which is reflected by the reduced eclosion rates of both the park ${ }^{25}$ heterozygous and

104 homozygous flies on control feces compared to the control flies on control feces (both $\mathrm{P}<0.0001$ ). In

105 agreement with pupation, a smaller amount of variation was due to the feces $(11.9 \%, P<0.0001)$ and

there was also a significant interaction between the fly genotype and the feces transfer, indicating that

107 there might be a specific effect of the fecal transfer in the park ${ }^{25}$ fly $(P=0.0042,1.4 \%$ of variation).

Regardless of the source of the fecal inoculum, both the heterozygous and homozygous park ${ }^{25}$

flies had reduced eclosion rates compared to the control flies, indicating that the park $^{25}$ genotype likely

has a reduced eclosion rate due to the park ${ }^{25}$ mutation (Fig. 2A). However, it appears that the park ${ }^{25}$

111 fecal transfer had an additional negative impact on the park ${ }^{25}$ flies. This feces-dependent differential

112 effect, based on fly genotype, of eclosion reduction due to the park ${ }^{25}$ feces becomes more apparent

113 when observing the number of pupae that failed to eclose. This measurement indicates that the control

114 flies did not have an increased number of failed eclosures $(P=0.1423)$, while the heterozygous and

115 homozygous park ${ }^{25}$ pupae did $(P=0.0023, P<0.0001$, respectively; Supplementary Fig. S2).

Further, the detrimental effects of the park ${ }^{25}$ feces on fly eclosion were of larger magnitude for

117 the homozygous park ${ }^{25}$ flies than other genotypes. When we calculated the percent eclosion rates of

118 each genotype on park ${ }^{25}$ feces relative to control feces, there was no difference in the eclosion rates of

119 the heterozygous mutants and control flies $(P=0.1211)$, but both genotypes had higher eclosion rates

120 than the park $^{25}$ homozygous flies ( $P<0.0001$ vs both, Fig. 2B). Further support of differential feces-

121 genotype interaction is provided by observing the eclosion rate over time. Supplementary Figure S3 
122 shows that the control flies experienced a reduction in eclosion due to park ${ }^{25}$ feces on day $10(P<$

123 0.0001), while heterozygous park ${ }^{25}$ flies had no significant reduction on any day in the experiment.

124 However, homozygous park ${ }^{25}$ flies experienced a reduction in eclosion due to park $^{25}$ feces on days 9,10

125 and $11(P=0.0001, P=0.0012, P=0.0005$, respectively $)$.

The whole-body microbiota varies between conventional PD model and control flies

Our observations suggest that the composition of park $^{25}$ and control fly microbiomes are

128 different and cause different developmental effects on the flies tested. Thus, as an extension of these

129 results we measured the bacterial microbiota of whole-body conventionally reared park ${ }^{25}$ and control

130 flies. The samples for sequencing were collected at a different time than the experiments above and,

131 because of the inconstant microbiota ${ }^{25}$, the sequencing results should not be conflated as measuring the

132 microbiota of the flies that deposited feces in the previous experiments. Sequencing of the V4 region of

133 the 16S rRNA gene revealed significant differences in the whole-body microbial communities of our

134 stocks. The most notable difference between the mutant and control populations was the presence of

135 the reproductive tract endosymbiont Wolbachia in the control flies (Supplementary Fig. S4 and

136 Supplementary Table S1); however, Wolbachia are not likely to be transferred between flies via

137 ingestion and therefore are not likely candidates for the effects observed with fecal transfer. After

138 Wolbachia were removed from the analysis (to focus on non-reproductive tract microorganisms), beta-

139 diversity metrics that factor microbial abundance reported significant differences in the microbiota

140 composition of the different fly stocks with fly genotype, but not with the sex of the flies (Fig. 3,

141 Supplementary Fig. S5, and Table 1). Also, there was not a significant genotype * sex interaction,

142 indicating that both males and females showed the same genotype-dependent changes in microbiota

143 composition (Table 1). Amplicon sequence variants assigned to the LAB (more abundant in controls) and

$144 \quad A A B$ (less abundant in controls) were significantly different in relative abundance between flies of

145 different genotypes (Supplementary Fig. S6). The decreased abundance of AAB in the control flies, 
which also bore Wolbachia, is consistent with previous reports that Wolbachia prevalence is negatively

147 associated with $A A B$ abundance ${ }^{26}$. Overall, the data reveal a consistent difference in the microbiota composition of Wolbachia-discordant control and park ${ }^{25}$ mutant flies that were reared side-by-side under conventional laboratory conditions.

\section{Axenic preparation of park $^{25}$ homozygous embryos has a dramatic effect on eclosion}

To determine if the different effects of fecal transfer from park $^{25}$ or control flies could be attributed to variation in the bacterial microbiota we measured eclosion rates in flies that were inoculated as sterile embryos with cultured feces from control and park ${ }^{25}$ adult flies. We observed two major differences between the different treatment approaches: fecal transfer vs fecal bacterial culture inoculation. First, the process of generating axenic embryos dramatically decreased the eclosion rates of homozygous, but not heterozygous, park ${ }^{25}$ mutants relative to controls. (Fig. $4, \mathrm{P}<0.0001$ ). Second, there was no effect related to feces source (control or park ${ }^{25}$ ) that were used to create the bacterial cultures on eclosion rate regardless of the genotype that received the bacterial culture. These results were not due to a limited number of homozygous pupae present in the tubes, as all experimental vials with park $^{25}$ pupae contained approximately $30 \%$ park $^{25}$ homozygous pupae, while the axenic experimental vials had the most pupae/vial (Supplementary Table S2). The most significant source of variation was fly genotype $(P<0.0001)$, accounting for $65 \%$ of the variation, with the bacterial status of the fly contributing only $0.23 \%$ to variation $(P=0.0082)$. No difference in the eclosion rates were observed between the axenic and the two gnotobiotic treatments regardless of fly genotype (all P > 0.52). Together, these results suggest that variation in the cultured bacteria in fly feces does not contribute to the variation in the eclosion rates observed in park ${ }^{25}$ mutant and control flies when reared on fly feces-seeded vials.

In contrast to whole-body flies, the major difference in the microbiota of fecal samples collected from park $^{25}$ mutant and control flies was attributed to microbial identity, not abundance (Table 2 where 
unweighted Unifrac, but not weighted Unifrac or Bray-Curtis distance metrics showed significant

171 variation in the community composition with host genotype; corresponding PCoA plots are in Figure 5

172 and Supplementary Figure S7. Analyzed feces were collected from independent experiments and,

173 because of the inconstant microbiota, the results cannot be directly compared to other experiments

174 here. Analysis of the fecal samples and siblings of the fecal donors revealed that the microbiota

175 composition varied with respect to both the host genotype and the sample type (fly or feces). For

176 example, the feces was dominated by an Enterococcus ASV (Fig. 5A) and the four AAB ASVs that were

177 most abundant in the flies (Fig. 3, Supplementary Table S3 and Supplementary Fig. S8) were detected 178 at very low levels. The low level of $A A B$ reads in the feces suggests that $A A B D N A$ in living or dead cells 179 persists poorly between the location of abundant bacterial cells in the flies and collection of $<24 \mathrm{~h}$ old 180 feces. Additionally or alternatively, Enterococcus cells may grow rapidly in the feces since there is little 181 evidence of their abundance in live flies, or Enterococcus DNA may survive gut transit well. Together, 182 these results suggest that the role of $\mathrm{P}$ generation defecation in establishing the $\mathrm{F} 1$ adult microbiota in the flies in our study, and perhaps flies generally, is incompletely understood.

Variation in the bacterial microbiota of $D$. melanogaster influences eclosion success in park ${ }^{25}$ mutants

To understand the extent to which bacterial microbiota of $D$. melanogaster influence eclosion success in homozygous and heterozygous park ${ }^{25}$ mutants, we compared eclosion rates of mono-

187 associated flies. Previous gnotobiotic experiments provided no direct evidence that variation in the 188 bacterial communities of the flies influenced fly eclosion in these park ${ }^{25}$ mutants. In contrast, fecal 189 transfer experiments suggested that microbiome changes did affect fly eclosion. Similar to our results 190 with cultured feces, we observed dramatically reduced eclosion in homozygous park ${ }^{25}$ flies compared to 191 heterozygous park ${ }^{25}$ flies (Fig. 6). Unlike with cultured feces, there were differences in fly eclosion rates 192 when they were axenic or colonized with a combination of 4 bacterial species cultured from flies in Ithaca, $\mathrm{NY}^{27}$ (heterozygous park ${ }^{25}: \mathrm{P}=0.0025$, homozygous park ${ }^{25}: \mathrm{P}<0.0001$ ) or individually with 
Acetobacter tropicalis (heterozygous park ${ }^{25}: \mathrm{P}=0.0088$, homozygous park ${ }^{25}: \mathrm{P}=0.0456$ ). Additionally, there was a significant fly genotype * bacterial treatment interaction (two-way ANOVA, $P<0.0001$ ): the combination and $A$. tropicalis treatments led to higher eclosion survival than the axenic treatment in park $^{25}$ homozygous flies; but lower survival than axenic flies for park ${ }^{25}$ heterozygous flies. Taken together, these results confirm that variation in the bacterial microbiota of park ${ }^{25}$ flies can contribute to variation in a key survival phenotype, eclosion success.

\section{Discussion}

There have been many studies showing alterations in the fecal microbiota of PD patients compared to control populations ${ }^{10}$. To our knowledge, there has only been a single study that has looked at the microbiota in a fly PD model $\left(P I N K 1^{B 9}\right)$ that also identified differences between the microbiome of the PD model and control flies ${ }^{21}$. Under condition-matched conventional rearing, our park $^{25}$ PD model fly microbiota was considerably different from the control fly (Fig. 3), with significant differences in the abundance of $A A B$ and $L A B$. Fly sex was not a determinant of variation in microbiota composition. Alterations in microbiota were observed in conventionally-reared flies and therefore factors such as inconstant exposure to or acquisition of distinct sets of microbes in the different vials from which samples were drawn could contribute to the observed effects; though the level of replication and matched rearing conditions of the flies suggests potential influences of host genotype on fly microbiota composition. Future experiments with gnotobiotic flies could conclusively rule out environmental effects but could be challenging because of the low survival rates of the homozygous $\operatorname{park}^{25}$ flies (Fig. 4).

Presence of the endosymbiont Wolbachia has been associated with lower counts of Acetobacter spp. in other flies ${ }^{28}$ and is positively correlated with worsening phenotypes in a fly model of Alzheimer's 
disease. In agreement with this, a recent report linked Wolbachia and neurodegenerative disease

218 severity in Drosophila by showing that administration of a Lactobacillus probiotic increased Acetobacter

219 abundance, lowered Wolbachia titers, and ameliorated Alzheimer's disease phenotypes ${ }^{29}$. Thus, the

220 presence of Wolbachia could be a factor contributing to differences between the microbiota of our

221 control and park ${ }^{25}$ flies. Our analysis identified that the Wolbachia status of our control and mutant

222 stocks was not congruent. In consideration of the fecal transfer, it is important to recognize that

223 Wolbachia are intracellular endosymbionts that are transmitted between generations via the germ line

224 and not fecal transfer ${ }^{22}$. This was validated by sequence analysis which demonstrated that Wolbachia

225 were not represented in the sequenced fecal samples, confirming their absence and irrelevance to

226 observed functional effects of the park ${ }^{25}$ fecal transfer (Fig. 5). Our data indicate that Acetobacter spp.

227 are reduced in our control flies both in diversity and abundance (Fig. 3 and Supplementary Fig. S6). The

228 data do raise the question if the differences in the eclosion of control and park ${ }^{25}$ flies is due to their

229 discordant Wolbachia status. While some influence on eclosion is possible, Wolbachia is unlikely to be

230 the sole contributor to this observed difference as PD phenotypes have been detected in another

231 laboratory using the same park ${ }^{25}$ mutant and a Wolbachia-concordant control strain $\left(^{5}\right.$, unpublished

232 data). The potential direct and indirect (through the microbiota) influence(s) Wolbachia has on park ${ }^{25}$

233 mutant development requires further analysis.

We used fecal transfers to assess whether the microbiota influences park ${ }^{25}$ mutant eclosion

success. Microbiota studies typically rear dechorionated embryos free of bacteria or with a defined

236 bacterial inoculum ${ }^{23}$, but we adopted an alternate fecal transfer approach for two reasons. First, fecal

237 transfers have successfully identified microbiota effects in other studies (e.g., ${ }^{30}$ ) and provided a

238 straightforward method to use in initial functional explorations. Second, as shown in Figures 4 and 6, the

239 viability of axenic and gnotobiotic park ${ }^{25}$ flies is very low, which makes this process extremely difficult

240 and impedes experimentation. We do not know the cause of this high mortality rate, but it appears to 
241 be related to the dechorionation process. Alternative approaches that avoid the dechorionation step

242 are available, but these approaches also have limitations. For example, while raising axenic fly stocks for

243 several generations after dechorionation the stocks are vulnerable to bacterial contamination, requiring

244 the use of antibiotics which can alter but not necessarily eliminate all colonizing microorganisms. One

245 successful recent approach fed bacteria to newly eclosed (and presumably bacteria-depleted) PINK1

246 mutant flies ${ }^{21}$, which might be a more high-throughput approach.

The exposure of hatching larvae to park $^{25}$ fly feces led to dramatically reduced fly eclosion

248 success than did exposure to control feces. Conversely, when we inoculated flies with cultured feces

249 there was no difference in the effect on fly eclosion. The difference in outcome between the two

250 experiments suggests that different effectors are transmitted, or possibly diluted, when the feces is

251 cultured first versus when it is directly deposited. We hypothesized that the fecal microbiota would

252 largely reflect the adult fly microbiota and that culturing feces versus direct deposition would lead to

253 similar outcomes. However, we detected no difference between the fecal microbiota of mutant and

254 control flies even though adult mutant and control flies had a different microbiota composition. In this

255 regard, the outcomes of the fecal transfer vs culture experiments were congruent: there was only a

256 difference in recipient phenotypes when there was a difference in the source's microbiota composition.

257 Altered phenotypes following the transfer of direct but not cultured feces could be also be due to an

258 effector that is abiotic or non-bacterial (e.g., fungal); or it may be that the culture step abates the effect.

259 Culture in standard laboratory medium may select for certain strains in ways that does not occur in the

260 fly diet, leading to differences in identity and abundance of key microbiota members. To address these

261 potential limitations, the bacterial mono-association experimental approach was critical to

262 understanding whether variation in bacterial microbiota can alter the eclosion success of park ${ }^{25}$ flies in a 263 genotype-dependent (heterozygous vs. homozygous) manner. 
We found that there is a functional consequence with the feces transfer, in that the park $^{25}$ feces

265 had a negative impact on pupation and eclosure on both the control and park ${ }^{25}$ flies; however, the 266 homozygous park ${ }^{25}$ flies appeared to be affected the most. It is possible that homozygous park ${ }^{25}$ flies

267 are more susceptible to park $^{25}$ feces due to their general weak state. It is established that park ${ }^{25} \mathrm{flies}$

268 have reduced mitochondrial function and deficiency in energy production ${ }^{5,7}$. Additionally, axenic flies

269 have disrupted insulin-like signaling and glucose regulation compared to microbiota-colonized

270 flies ${ }^{24,31,32}$. Therefore, axenic homozygous park ${ }^{25}$ flies may have compounding, additive deficiencies that

271 reduce their ATP production during eclosion, which is likely a high energy-requiring process. In support

272 of this idea, $34.9 \%$ of all axenic park ${ }^{25}$ pupae were found dead and stuck in the process of eclosing,

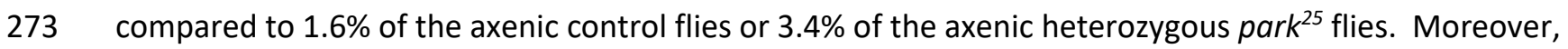

274 when the park ${ }^{25}$ homozygous flies had two fecal-derived gnotobiotic treatments, the rate of being stuck

275 in eclosure reduced to $15.9 \%$ with the control bacteria and $13.4 \%$ with the park ${ }^{25}$ bacteria. This reduced

276 rate of incomplete eclosion in the gnotobiotic populations might have masked the negative effect of the

277 park ${ }^{25}$ bacteria on eclosure in these flies compared to the axenic controls, however, since both

278 gnotobiotic groups had similar reductions in getting stuck, this is unlikely.

Despite the large number of studies showing an altered fecal microbiome in PD patients, there

280 have been very few studies demonstrating that the PD microbiome has functional consequences. The

281 most compelling functional study utilized PD model mice that had a fecal transplant from PD and

282 healthy control patients. The PD microbiome transplant mice showed an increase in motor dysfunction

283 and alpha-synuclein aggregation ${ }^{11}$. Our fecal transfer experiments, like another recent Drosophila PD

284 model functional microbiome study ${ }^{21}$, did not directly manipulate the microbiome to a specifically-

285 defined microbiota composition but we do use mono-association experiments to demonstrate species-

286 specific influences of the associated microorganisms. Our study is the first to indicate that there might

287 be a specific microbiota * fly genotype effect that might also be occurring with the PD microbiome and 
288 homozygous park ${ }^{25}$ flies. This type of specific microbiota * fly genotype interaction is known to happen

289 with Wolbachia ${ }^{33}$. This study adds to the small group of publications that indicate that the altered PD

290 microbiome negatively affects biological processes in the host, which has major implications for PD

291 patients.

292

293 Methods

294

Drosophila Stocks and Maintenance

295

Mutant, park $^{25}$, flies were provided by Dr. Leo Pallanck at the University of Washington. This

mutant stock was derived from $w^{1118}$ control flies, which were obtained from the Bloomington

297 Drosophila Stock Center (Indiana University). In all experiments, $w^{1118}$ flies were used as the control for

298 the park ${ }^{25}$ flies. The park ${ }^{25}$ stock in our laboratory has been backcrossed with the $w^{1118}$ stock so that all

299 chromosomes are from the $w^{1118}$ background. The park ${ }^{25}$ chromosome is balanced over the TM6C

300 balancer, allowing for identification of homozygous and heterozygous flies through use of the Tubby

301 gene phenotype. All fly stocks were raised on standard cornmeal-molasses diet at $25^{\circ} \mathrm{C}$ in a $14 / 10$-hour

302 light cycle.

303 Fecal transfer

In each of three separate experiments, five separate food vials for each fly genotype were

305 seeded with forty males of that genotype to allow flies to deposit their feces on the food. Males were

306 used so that no embryos were laid on the food. All males were over the age of three days to ensure that

307 they had an established microbiome ${ }^{34}$. A random mix of both homozygous and heterozygous park ${ }^{25}$

308 male flies were used as the fecal donors for the $\operatorname{park}^{25}$ feces. Four days post-seeding, 60 embryos of the 
specific genotype were placed on the feces-prepared food. The embryos were collected and counted as

310 described below.

\section{Embryo collection}

Fly stocks (control and park ${ }^{25}$-heterozygous and homozygous) were placed in square

313 polypropylene fly bottles with a molasses "puck" as the lid. The molasses puck was a $35 \mathrm{~mm}$ petri dish

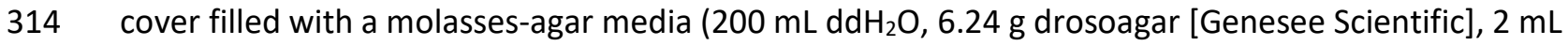

315 Tegosept, and $50 \mathrm{~mL}$ of molasses). The bottles were stored upside down, so the puck was at the bottom,

316 while the top of the bottle had small holes for air transfer. Yeast paste was put on the inside of the

317 bottle to help stimulate oogenesis. Flies were allowed to lay their embryos for $<24$ hours at $25^{\circ} \mathrm{C}$.

318 Embryo collection was performed by wetting a paintbrush with $\mathrm{ddH}_{2} \mathrm{O}$ and carefully brushing the

319 embryos from the molasses-agar. The embryos were then washed off the puck directly into a $1.5 \mathrm{~mL}$

320 centrifuge tube and were rinsed with $\mathrm{ddH}_{2} \mathrm{O}$ for a total of three washes. After washing, the embryos

321 were pipetted into a glass spot plate in one of the three wells. Under a stereo microscope, 60 embryos

322 were counted and placed in one of the empty wells with $<0.5 \mathrm{ml}$ water. A fine-tipped paintbrush was

323 used to paint the 60 embryos onto the surface of the fecal-prepared food. A different paintbrush was

324 used for each fly genotype to prevent bacterial transfer between genotypes during embryo deposition.

325 The embryos were collected for six days, with new parental fly populations being introduced every two

326 days to produce three biological replicates with each biological replicate having two technical replicates.

\section{Axenic and gnotobiotic experiments}

We reared flies with bacteria cultured directly from fly feces beginning with axenic fly embryos.

329 Axenic embryos were derived as described previously ${ }^{23}$. Briefly, control and park ${ }^{25}$ embryos were

330 collected as above and suspended in a $0.6 \%$ sodium hypochlorite solution for 2.5 minutes. These

331 embryos were then transferred to fresh $0.6 \%$ sodium hypochlorite solution in a sterile hood to 
332 dechorionate the embryos. The sterile, dechorionated embryos were collected with a sterile paintbrush 333 and approximately 60 embryos were brushed onto sterile food. These embryos were either maintained 334 as axenic or inoculated with $5 \times 10^{5}$ CFUs from control or park ${ }^{25}$ fecal bacterial cultures, or from individual 335 bacterial strains, including, Lactobacillus brevis, Lactiplantibacillus plantarum, Acetobacter pomorum, or 336 Acetobacter tropicalis.

To produce the fecal bacterial cultures, feces were collected from the park ${ }^{25}$ and control fly 338 embryo collection bottles by scraping the feces off with a sterile toothpick to inoculate Luria-Bertani (LB) 339 and modified deMan-Rogosa-Sharpe (mMRS) medium. These cultures were grown at $30^{\circ} \mathrm{C}$ with aeration 340 for 16 hrs. To preferentially cultivate aerotolerant microbes, separate MRS cultures were grown in 341 loosely capped tubes with no shaking for $16 \mathrm{hrs}$. To generate the microbiome inoculum, each culture 342 was normalized to $10^{7} \mathrm{~mL}^{-1}$, combined in equivalent ratios, and $50 \mu$ l containing $5 \times 10^{5}$ total CFUs was 343 used to inoculate the sterile embryos. Control axenic embryos were collected each day for four days 344 with a minimum of eight tubes/day, while the axenic park ${ }^{25}$ embryos were collected each day for five 345 days with a minimum of 28 tubes/day. The bacterial culture embryos were collected each day for four 346 days with a minimum of 10 tubes/day for control embryos or 14 tubes/day for the park ${ }^{25}$ embryos.

To confirm axenic flies were truly bacteria-free, pools of five whole-body adult axenic flies from 348 each axenic control vial were homogenized the end of each experiment and cultured on LB and 349 duplicate MRS plates (one incubated with standard atmospheric conditions, one in microoxic conditions 350 in a sealed, $\mathrm{CO}_{2}$-flooded chamber) at $30^{\circ} \mathrm{C}$. If $>10 \mathrm{CFU} / \mathrm{fly}$ were detected, those flies were deemed non351 axenic and removed from the analysis.

\section{Pupation and eclosure measurements}

354 Tubby mutation on the TM6C balancer chromosome present in the heterozygous park ${ }^{25}$ flies, 
355 homozygous and heterozygous park ${ }^{25}$ flies were differentiated. Although analyzed separately, these

356 two pupal populations account for the full 60 park ${ }^{25}$ embryos painted in the fecal transfer experiments.

357 Individual fly eclosion was quantified on days 9, 10, 11 and 12 post-embryo collection.

$16 \mathrm{~S}$ sequencing and analysis

We prepared and analyzed DNA samples for 16S rRNA marker gene analysis as done previously ${ }^{26,28}$. Sequencing libraries were prepared by extracting DNA from pools of 10 flies using the

361 Zymo Quick-DNA fecal/soil microbe kit (D6011, Zymo, Irvine, CA). Then, the V4 region of the extracted

362 DNA was amplified and sequenced using a dual-barcoding method described by Kozich ${ }^{35}$, with the 363 exception of substituting Accuprime PFX DNA polymerase reagents for Accuprime PFX Supermix. The 364 Invitrogen SequalPrep Normalization kit was used to normalize samples into pools of 96 samples (in 365 some cases, the samples were normalized as part of a pool with samples not published in this study).

366 Then, fragments in the size range of 250-450 nucleotides were size-selected using a BluePippin (BYU

367 DNA Sequencing Center). Finally, samples in this study were sequenced on partial lanes of a MiSeq using 368500 cycle chemistry (paired-end 2 x 250, BioDesign Institute at Arizona State University)

370 scores, denoised and dereplicated using DADA2 ${ }^{37}$ to call individual amplicon sequence variants (ASVs), 371 and taxonomy was assigned using the GreenGenes classifier ${ }^{38,39}$. To enable the calculation of Unifrac 372 beta-diversity metrics ${ }^{40,41}$, a phylogeny of all ASVs was constructed ${ }^{42}$ based on mafft alignment ${ }^{43}$. For 373 some analyses, Wolbachia reads were pre-filtered out so that reproductive tract symbionts were not 374 included in the analysis. Before performing beta-diversity analyses, samples were normalized to varying 375 read thresholds that maximized the number of reads per sample and the number of samples retained:

376350 (Fig. 3), 3000 (Fig. 5), and 399 (Supplementary Fig. S8). Permutational multivariate analysis of 377 variance (PERMANOVA) of Bray Curtis distances and of unweighted and weighted Unifrac distances 
378 were used to test for host genotype and sex-dependent variation in microbiota composition ${ }^{44}$. We also

379 used Analysis of Composition of Microbiomes (ANCOM) to test for differences in the abundances of

380 specific individual or groups of ASVs $^{45}$.

One sample was removed from Figure $\mathbf{3}$ analyses because it was almost exclusively

382

enterococcus. Removing it did not change the significance of any comparisons but did reduce noise.

383

Analyses of the data that include this sample are presented in Supplementary Figure S2.

\section{Development Statistical Analysis}

Statistical analyses were performed by using One-Way and Two-Way ANOVA, with post-hoc

Tukey's, Sidak's, or Dunnett's tests to determine differences between the arcsin transformed

387

percentages of each group by GraphPad Prism 9. All other data analysis was in RStudio version 1.3.1093

using $\mathrm{R}$ version 3.6 .3 or the terminal. All graphs display the mean \pm the standard error of the mean.

Details on each test performed and their results are presented in the results section or legends.

391 Author contributions

392 Study conception and design: G.B.C.; Study supervision: G.B.C., J.M.C., and S.B.K.; Data collection: J.P.-C.,

393 D.R.H., T.B.C., Z.S.P., S.A.T.; Data and statistical analyses: G.B.C., E.M.M., and J.M.C.; Writing and

394 revision: G.B.C., J.M.C. and S.B.K.

\section{Data availability}

397 The reads are publicly available at the NCBI SRA under ACCESSION FORTHCOMING. 
399 Code availability

400 Code used for this study can be obtained by contacting the corresponding author.

401

402 Competing interests

403 The authors declare no competing interests. 


\section{References}

4051 Statistics - Parkinson's Foundation, < 1 https://www.parkinson.org/Understanding-

$406 \quad$ Parkinsons/Statistics> (2020).

4072 Hernandez, D. G., Reed, X. \& Singleton, A. B. Genetics in Parkinson disease: Mendelian versus

408 non-Mendelian inheritance. J Neurochem 139 Suppl 1, 59-74, doi:10.1111/jnc.13593 (2016).

4093 Lücking, C. B. et al. Association between early-onset Parkinson's disease and mutations in the parkin gene. N Engl J Med 342, 1560-1567, doi:10.1056/NEJM200005253422103 (2000).

4114 Chambers, R. P. et al. Nicotine increases lifespan and rescues olfactory and motor deficits in a

412 Drosophila model of Parkinson's disease. Behavioural brain research 253, 95-102,

413 doi:10.1016/j.bbr.2013.07.020 (2013).

4145 Greene, J. C. et al. Mitochondrial pathology and apoptotic muscle degeneration in Drosophila

415 parkin mutants. Proc Natl Acad Sci U S A 100, 4078-4083, doi:10.1073/pnas.0737556100 (2003).

4166 Whitworth, A. J. et al. Increased glutathione S-transferase activity rescues dopaminergic neuron 417 loss in a Drosophila model of Parkinson's disease. Proc Natl Acad Sci U S A 102, 8024-8029, 418 doi:10.1073/pnas.0501078102 (2005).

4197 Cackovic, J. et al. Vulnerable Parkin Loss-of-Function Drosophila Dopaminergic Neurons Have 420 Advanced Mitochondrial Aging, Mitochondrial Network Loss and Transiently Reduced

421 Autophagosome Recruitment. Front Cell Neurosci 12, 39, doi:10.3389/fncel.2018.00039 (2018).

4228 Hewitt, V. L. \& Whitworth, A. J. Mechanisms of Parkinson's Disease: Lessons from Drosophila. $423 \quad$ Curr Top Dev Biol 121, 173-200, doi:10.1016/bs.ctdb.2016.07.005 (2017).

4249 Fasano, A., Visanji, N. P., Liu, L. W., Lang, A. E. \& Pfeiffer, R. F. Gastrointestinal dysfunction in 425 Parkinson's disease. Lancet Neurol 14, 625-639, doi:10.1016/S1474-4422(15)00007-1 (2015). 
42610 Boertien, J. M., Pereira, P. A. B., Aho, V. T. E. \& Scheperjans, F. Increasing Comparability and 427 Utility of Gut Microbiome Studies in Parkinson's Disease: A Systematic Review. J Parkinsons Dis 9, S297-S312, doi:10.3233/JPD-191711 (2019).

42911 Sampson, T. R. et al. Gut Microbiota Regulate Motor Deficits and Neuroinflammation in a Model of Parkinson's Disease. Cell 167, 1469-1480.e1412, doi:10.1016/j.cell.2016.11.018 (2016).

43112 Wong, C. N., Ng, P. \& Douglas, A. E. Low-diversity bacterial community in the gut of the fruitfly Drosophila melanogaster. Environ Microbiol 13, 1889-1900, doi:10.1111/j.14622920.2011.02511.x (2011).

13 Chandler, J. A., Lang, J. M., Bhatnagar, S., Eisen, J. A. \& Kopp, A. Bacterial communities of diverse Drosophila species: ecological context of a host-microbe model system. PLoS genetics 7, e1002272, doi:10.1371/journal.pgen.1002272 (2011).

43714 Staubach, F., Baines, J. F., Kunzel, S., Bik, E. M. \& Petrov, D. A. Host species and environmental 438 effects on bacterial communities associated with Drosophila in the laboratory and in the natural environment. PLoS One 8, e70749, doi:10.1371/journal.pone.0070749 (2013).

44015 Chaston, J. M., Dobson, A. J., Newell, P. D. \& Douglas, A. E. Host Genetic Control of the 441 Microbiota Mediates the Drosophila Nutritional Phenotype. Appl Environ Microbiol 82, 671-679, doi:10.1128/AEM.03301-15 (2016).

44316 Carmody, R. N. et al. Diet dominates host genotype in shaping the murine gut microbiota. Cell Host Microbe 17, 72-84, doi:10.1016/j.chom.2014.11.010 (2015).

44517 Goodrich, J. K. et al. Human genetics shape the gut microbiome. Cell 159, 789-799, 446 doi:10.1016/j.cell.2014.09.053 (2014).

44718 Winans, N. J. et al. A genomic investigation of ecological differentiation between free-living and Drosophila-associated bacteria. Mol Ecol 26, 4536-4550, doi:10.1111/mec.14232 (2017). 
44919 Pais, I. S., Valente, R. S., Sporniak, M. \& Teixeira, L. Drosophila melanogaster establishes a species-specific mutualistic interaction with stable gut-colonizing bacteria. PLoS biology 16, e2005710, doi:10.1371/journal.pbio.2005710 (2018).

45220 Gould, A. L. et al. Microbiome interactions shape host fitness. Proc Natl Acad Sci U S A 115, $453 \quad$ E11951-E11960, doi:10.1073/pnas.1809349115 (2018).

45421 Xu, Y. et al. EGCG ameliorates neuronal and behavioral defects by remodeling gut microbiota 455 and TotM expression in Drosophila models of Parkinson's disease. FASEB J 34, 5931-5950, 456 doi:10.1096/fj.201903125RR (2020).

45722 Werren, J. H., Baldo, L. \& Clark, M. E. Wolbachia: master manipulators of invertebrate biology. $458 \quad$ Nat Rev Microbiol 6, 741-751, doi:10.1038/nrmicro1969 (2008).

$45923 \quad$ Koyle, M. L. et al. Rearing the Fruit Fly Drosophila melanogaster Under Axenic and Gnotobiotic $460 \quad$ Conditions. Journal of visualized experiments : JoVE, doi:10.3791/54219 (2016).

46124 Shin, S. C. et al. Drosophila microbiome modulates host developmental and metabolic homeostasis via insulin signaling. Science 334, 670-674, doi:10.1126/science.1212782 (2011).

46325 Wong, A. C., Chaston, J. M. \& Douglas, A. E. The inconstant gut microbiota of Drosophila species 464 revealed by 16S rRNA gene analysis. ISME J 7, 1922-1932, doi:10.1038/ismej.2013.86 (2013).

46526 Walters, A. W. et al. The microbiota influences the Drosophila melanogaster life history strategy. $466 \quad$ Mol Ecol 29, 639-653, doi:10.1111/mec.15344 (2020).

$46727 \quad$ Newell, P. D. et al. In vivo function and comparative genomic analyses of the Drosophila gut 468 microbiota identify candidate symbiosis factors. Front Microbiol 5, 576, 469 doi:10.3389/fmicb.2014.00576 (2014).

47028 Rudman, S. M. et al. Microbiome composition shapes rapid genomic adaptation of. Proc Natl $471 \quad$ Acad Sci U S A 116, 20025-20032, doi:10.1073/pnas.1907787116 (2019). 
47229 Tan, F. H. P. et al. Lactobacillus probiotics improved the gut microbiota profile of a Drosophila 473 melanogaster Alzheimer's disease model and alleviated neurodegeneration in the eye. Benef $474 \quad$ Microbes 11, 79-89, doi:10.3920/BM2019.0086 (2020).

47530 Zare, A., Johansson, A. M., Karlsson, E., Delhomme, N. \& Stenberg, P. The gut microbiome 476 participates in transgenerational inheritance of low-temperature responses in Drosophila 477 melanogaster. FEBS Lett 592, 4078-4086, doi:10.1002/1873-3468.13278 (2018).

47831 Ridley, E. V., Wong, A. C., Westmiller, S. \& Douglas, A. E. Impact of the resident microbiota on 479 the nutritional phenotype of Drosophila melanogaster. PLoS One 7, e36765, 480 doi:10.1371/journal.pone.0036765 (2012).

48132 Storelli, G. et al. Lactobacillus plantarum promotes Drosophila systemic growth by modulating 482 483

48433 Simhadri, R. K. et al. The Gut Commensal Microbiome of Drosophila melanogaster Is Modified by 485 486 34 Blum, J. E., Fischer, C. N., Miles, J. \& Handelsman, J. Frequent replenishment sustains the 487 488 beneficial microbiome of Drosophila melanogaster. mBio 4, e00860-00813, dual-index sequencing strategy and curation pipeline for analyzing amplicon sequence data on the MiSeq Illumina sequencing platform. Appl Environ Microbiol 79, 5112-5120, doi:10.1128/AEM.01043-13 (2013). Bolyen, E. et al. Reproducible, interactive, scalable and extensible microbiome data science using QIIME 2. Nat Biotechnol 37, 852-857, doi:10.1038/s41587-019-0209-9 (2019). 
49537 Callahan, B. J. et al. DADA2: High-resolution sample inference from Illumina amplicon data.

$496 \quad$ Nature methods 13, 581-583, doi:10.1038/nmeth.3869 (2016).

$49738 \quad$ McDonald, D. et al. An improved Greengenes taxonomy with explicit ranks for ecological and

498 evolutionary analyses of bacteria and archaea. ISME J 6, 610-618, doi:10.1038/ismej.2011.139

$499 \quad$ (2012).

50039 Bokulich, N. A. et al. Optimizing taxonomic classification of marker-gene amplicon sequences

501 with QIIME 2's q2-feature-classifier plugin. Microbiome 6, 90, doi:10.1186/s40168-018-0470-z

$502 \quad$ (2018).

$50340 \quad$ Lozupone, C. \& Knight, R. UniFrac: a new phylogenetic method for comparing microbial

504 communities. Appl Environ Microbiol 71, 8228-8235, doi:10.1128/AEM.71.12.8228-8235.2005

$505 \quad$ (2005)

50641 Lozupone, C. A., Hamady, M., Kelley, S. T. \& Knight, R. Quantitative and qualitative beta diversity

507 measures lead to different insights into factors that structure microbial communities. Appl

$508 \quad$ Environ Microbiol 73, 1576-1585, doi:10.1128/AEM.01996-06 (2007).

50942 Price, M. N., Dehal, P. S. \& Arkin, A. P. FastTree 2--approximately maximum-likelihood trees for

$510 \quad$ large alignments. PLoS One 5, e9490, doi:10.1371/journal.pone.0009490 (2010).

$51143 \quad$ Katoh, K., Misawa, K., Kuma, K. \& Miyata, T. MAFFT: a novel method for rapid multiple sequence

512 alignment based on fast Fourier transform. Nucleic Acids Res 30, 3059-3066,

513 doi:10.1093/nar/gkf436 (2002).

51444 Oksanen, J. et al. (Retrieved from https://CRAN.R-project.org/package=vegan, 2018).

$51545 \quad$ Mandal, S. et al. Analysis of composition of microbiomes: a novel method for studying microbial

516 composition. Microb Ecol Health Dis 26, 27663, doi:10.3402/mehd.v26.27663 (2015). 


\section{Tables}

519 Table 1. Genotype and sex-dependent differences in microbiota composition of control and park ${ }^{25}$

520 mutants.

521 Effects of fly genotype (G), fly sex (S), fly vial (V), and the G* S interaction (GS) are shown along with

522 residuals $(\mathrm{R})$ and totals $(\mathrm{T})$ as determined by PERMANOVA. PERMANOVA values are degrees of freedom

523 (df), sum of squares (SS), mean squares (MS), $F$ statistic $(F), R^{2}$ value $\left(R^{2}\right)$, and $P$-value $(P)$.

524

\begin{tabular}{|l|r|l|l|l|l|l|l|l|l|l|l|l|l|l|l|l|}
\hline \multicolumn{1}{|c|}{} & \multicolumn{5}{|c|}{ Weighted Unifrac } & \multicolumn{5}{|c|}{ Unweighted Unifrac } & \multicolumn{5}{c|}{ Bray Curtis } \\
\hline & df & SS & MS & F & $R^{2}$ & P & SS & MS & F & $R^{2}$ & P & SS & MS & F & $R^{2}$ & P \\
\hline G & 2 & 0.37 & 0.19 & 72.18 & 0.71 & 0.001 & 0.20 & 0.10 & 6.12 & 0.21 & 0.001 & 6.71 & 3.36 & 111.90 & 0.79 & 0.001 \\
\hline S & 1 & 0.00 & 0.00 & 1.38 & 0.01 & 0.24 & 0.02 & 0.02 & 1.25 & 0.02 & 0.29 & 0.04 & 0.04 & 1.33 & 0.00 & 0.25 \\
\hline V & 3 & 0.04 & 0.01 & 5.02 & 0.07 & 0.002 & 0.04 & 0.01 & 0.90 & 0.05 & 0.54 & 0.47 & 0.16 & 5.27 & 0.06 & 0.001 \\
\hline GS & 2 & 0.00 & 0.00 & 0.80 & 0.01 & 0.49 & 0.02 & 0.01 & 0.73 & 0.02 & 0.70 & 0.02 & 0.01 & 0.41 & 0.00 & 0.79 \\
\hline R & 41 & 0.11 & 0.00 & 0.20 & & & 0.67 & 0.02 & 0.70 & & & 1.23 & 0.03 & 0.15 & & \\
\hline T & 49 & 0.53 & 1.00 & & & & 0.96 & 1.00 & & & & 8.48 & 1.00 & & & \\
\hline
\end{tabular}

525 
527 mutants.

528 Effects of fly genotype (G), fly vial (V), residuals (R), and totals $(T)$ as determined by PERMANOVA.

529 PERMANOVA values are degrees of freedom (df), sum of squares (SS), mean squares (MS), F statistic (F),

$530 \quad R^{2}$ value $\left(R^{2}\right)$, and $P$-value $(P)$.

531

\begin{tabular}{|l|l|l|l|l|l|l|l|l|l|l|l|l|l|l|l|l|}
\hline & & \multicolumn{4}{|c|}{ Weighted Unifrac } & \multicolumn{5}{c|}{ Unweighted Unifrac } & \multicolumn{5}{|c|}{ Bray-Curtis } \\
\hline & df & SS & MS & F & $R^{2}$ & P & SS & MS & F & $R^{2}$ & P & SS & MS & F & $R^{2}$ & P \\
\hline G & 1 & 0.02 & 0.02 & 2.34 & 0.09 & 0.061 & 0.38 & 0.38 & 2.60 & 0.10 & 0.003 & 0.24 & 0.24 & 1.82 & 0.08 & 0.10 \\
\hline V & 10 & 0.11 & 0.01 & 1.24 & 0.46 & 0.26 & 1.54 & 0.15 & 1.05 & 0.42 & 0.36 & 1.16 & 0.12 & 0.89 & 0.39 & 0.61 \\
\hline R & 12 & 0.10 & 0.01 & 0.45 & & & 1.76 & 0.15 & 0.48 & & & 1.57 & 0.13 & 0.53 & & \\
\hline T & 23 & 0.23 & 1.00 & & & & 3.69 & 1.00 & & & & 2.96 & 1.00 & & & \\
\hline
\end{tabular}

532 


\section{Figures}

534 Figure 1. Feces from park $^{25}$ flies reduces total pupation rate.

535 Total pupation rates were calculated in control, heterozygous (Het) park ${ }^{25}$ and homozygous (Hom)

536 park $^{25}$ flies from the 60 embryos that were placed on food that had control or park ${ }^{25}$ feces present. Data

537 are presented as mean and SEM. Asterisks represent the results of a post-hoc Sidak's multiple

538 comparisons test comparing the two feces groups within each fly genotype ( $* * * *=\mathrm{P}<0.0001)$. Results

539 are from 45 separate vials in each group.

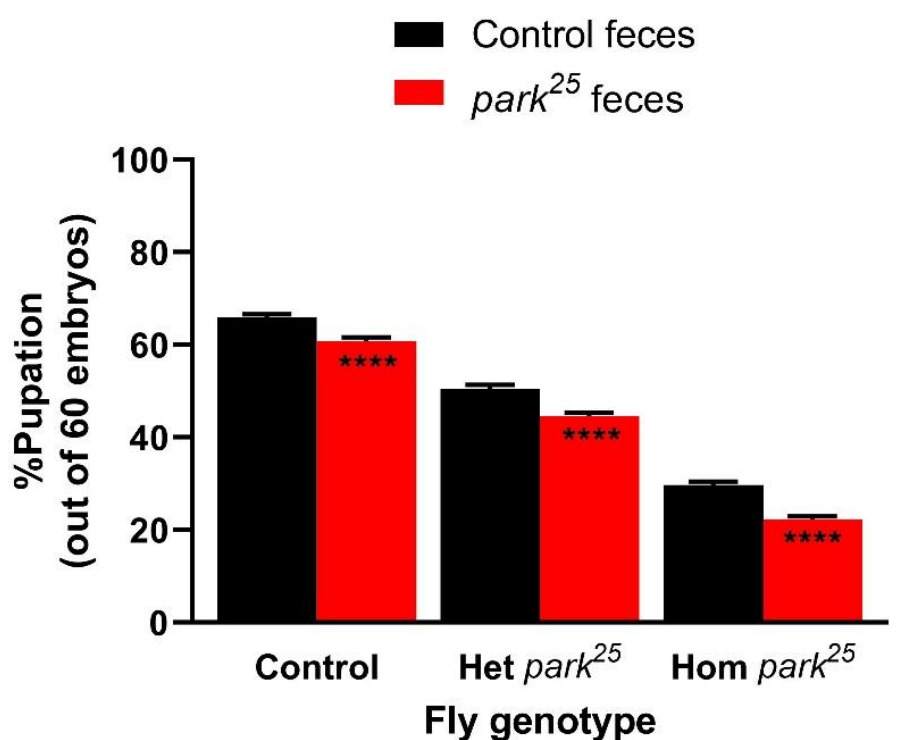


541 Figure 2. Eclosion rates are reduced in all fly genotypes but more in homozygous park ${ }^{25}$ flies with

542 park $^{25}$ feces transfer.

543 A) Total eclosion rates were determined in control, heterozygous (Het) park ${ }^{25}$ and homozygous (Hom)

544 park $^{25}$ flies based on the total number of pupae for each genotype. B) The relative eclosion reduction

545 caused by the park $^{25}$ fecal transfer, calculated as a percentage of the control feces eclosion, was

546 determined. Data are presented as mean and SEM. Asterisks represent the results of a post-hoc

547 Tukey's multiple comparisons test. Asterisks inside the bars compare the two feces groups within each

548 fly genotype. ${ }^{*}=\mathrm{P}<0.05$ and $* * * *=\mathrm{P}<0.0001$. Results are from 45 separate vials in each group.

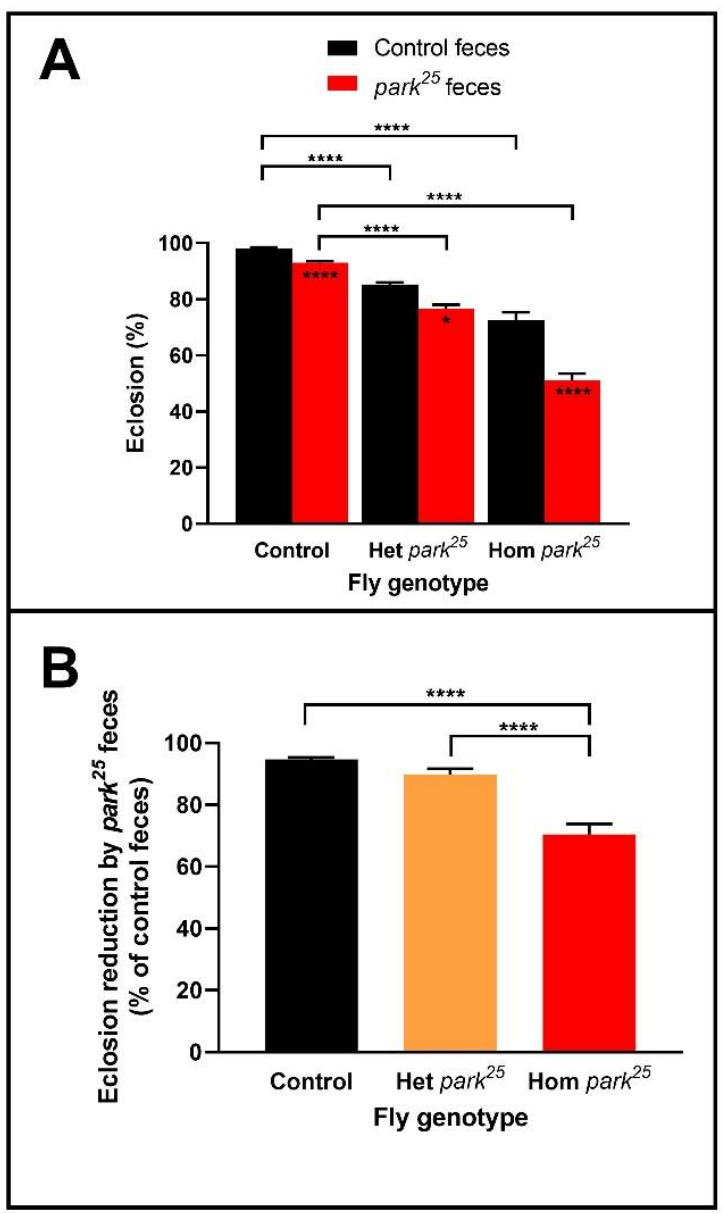


Figure 3. The microbiota of control and park $^{25}$ flies.

551 A) Taxon plot of control and park ${ }^{25}$ flies, separated by sex. Mutants of park $^{25}$ were distinguished as

552 homozygotes and heterozygotes based on the presence of the Tubby marker. Bars represent distinct

553 ASVs. The legend shows the lowest taxonomic level that was assigned to each ASV. B) Principal

554 coordinates plot, showing the first two coordinates calculated from a weighted Unifrac distance matrix.
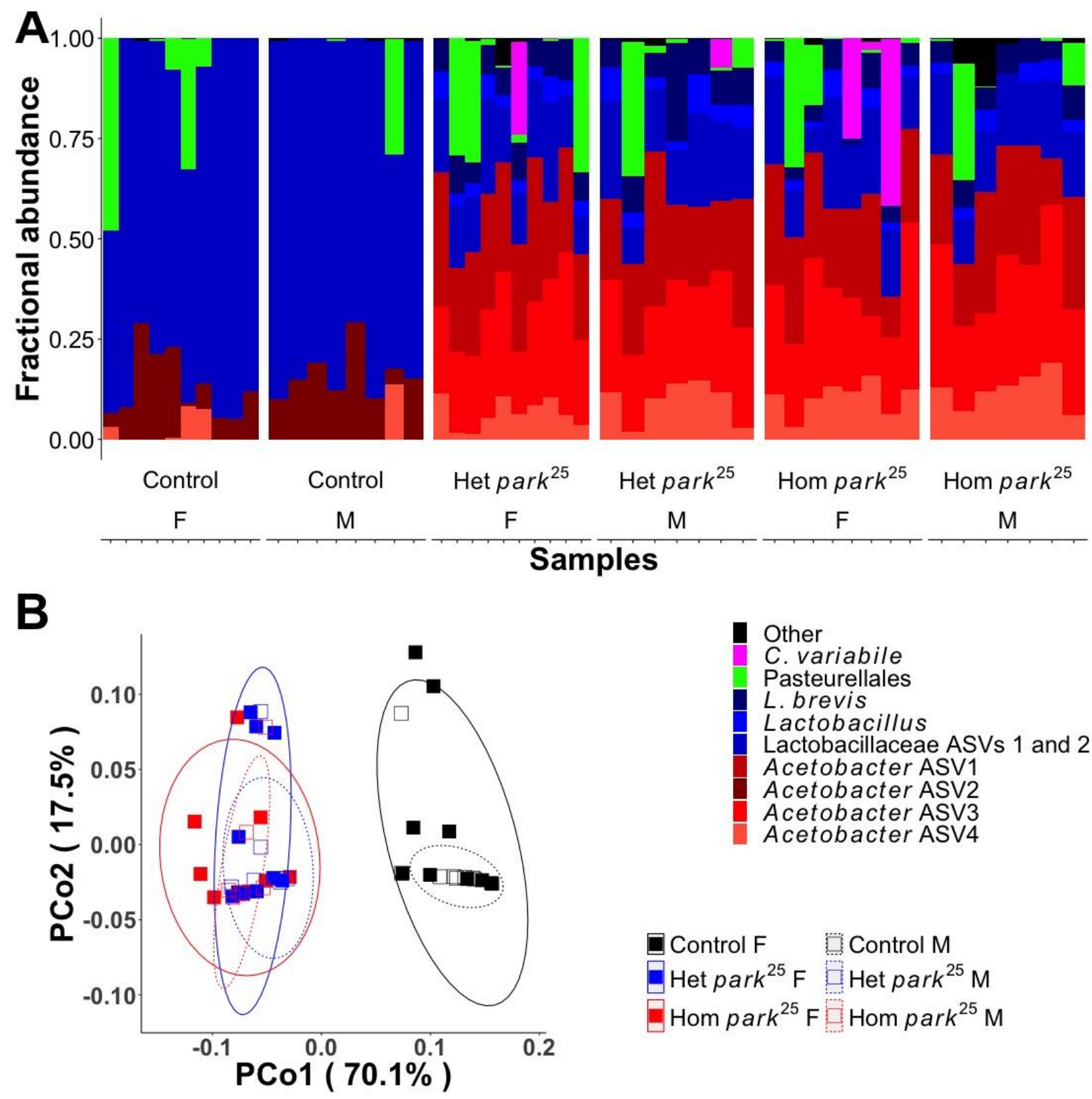

Other

C. variabile

Pasteurellales

L. brevis

Lactobacillus

Lactobacillaceae ASVs 1 and 2

Acetobacter ASV1

Acetobacter ASV2

Acetobacter ASV3

Acetobacter ASV4 
557 Embryos from park $^{25}$ and control flies were made axenic and gnotobiotic for feces-derived bacteria from 558 park $^{25}$ or control flies. Pupae count and eclosion was recorded from each vial. Heterozygous (Het) park ${ }^{25}$ 559 pupae were differentiated from the homozygous (Hom) park ${ }^{25}$ pupae by the presence of the Tubby 560 marker on the TM6C balancer chromosome. Data are presented as mean and SEM. Post-hoc Tukey's 561 analysis results are shown: ${ }^{* * *}=\mathrm{P}<0.0001$. Sample (number of vials) sizes: Control (axenic $=40$, 562 control feces $=41$, park $^{25}$ feces $\left.=42\right)$, Het \& Hom park ${ }^{25}$ (axenic $=202$, control feces $=63$, park $^{25}$ feces $=$ $56359)$.

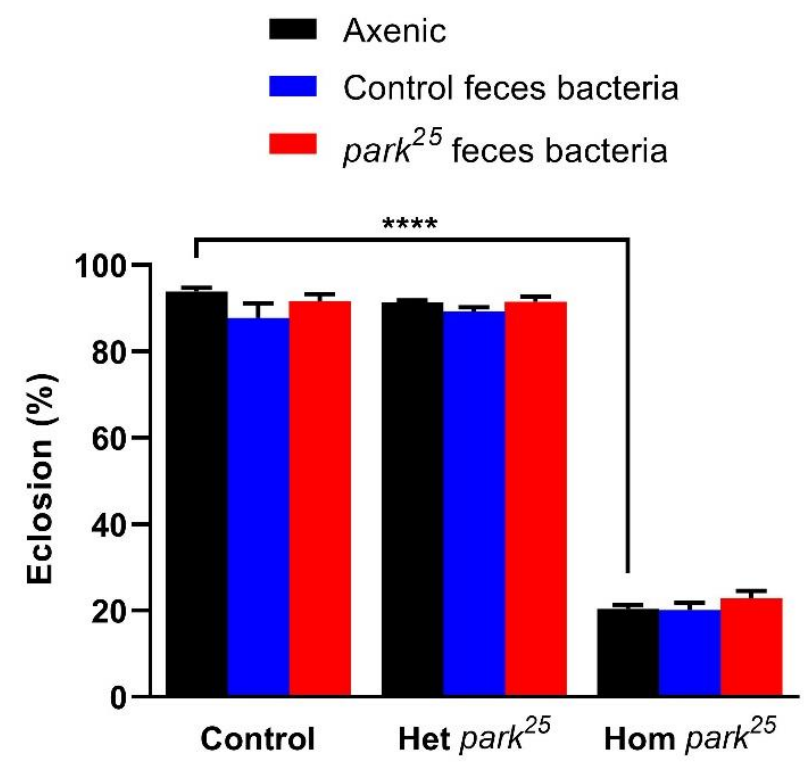

Fly genotype 
Figure 5. The microbiota of feces from control and park ${ }^{25}$ flies.

Fecal samples were collected from male controls and a mixture of heterozygous and homozygous male

567 park ${ }^{25}$ flies. A) A taxon plot with bars representing distinct ASVs. The legend shows the lowest taxonomic

568 level that was assigned to each ASV. B) Principal coordinates plot, showing the first two coordinates

569 calculated from a weighted Unifrac distance matrix.

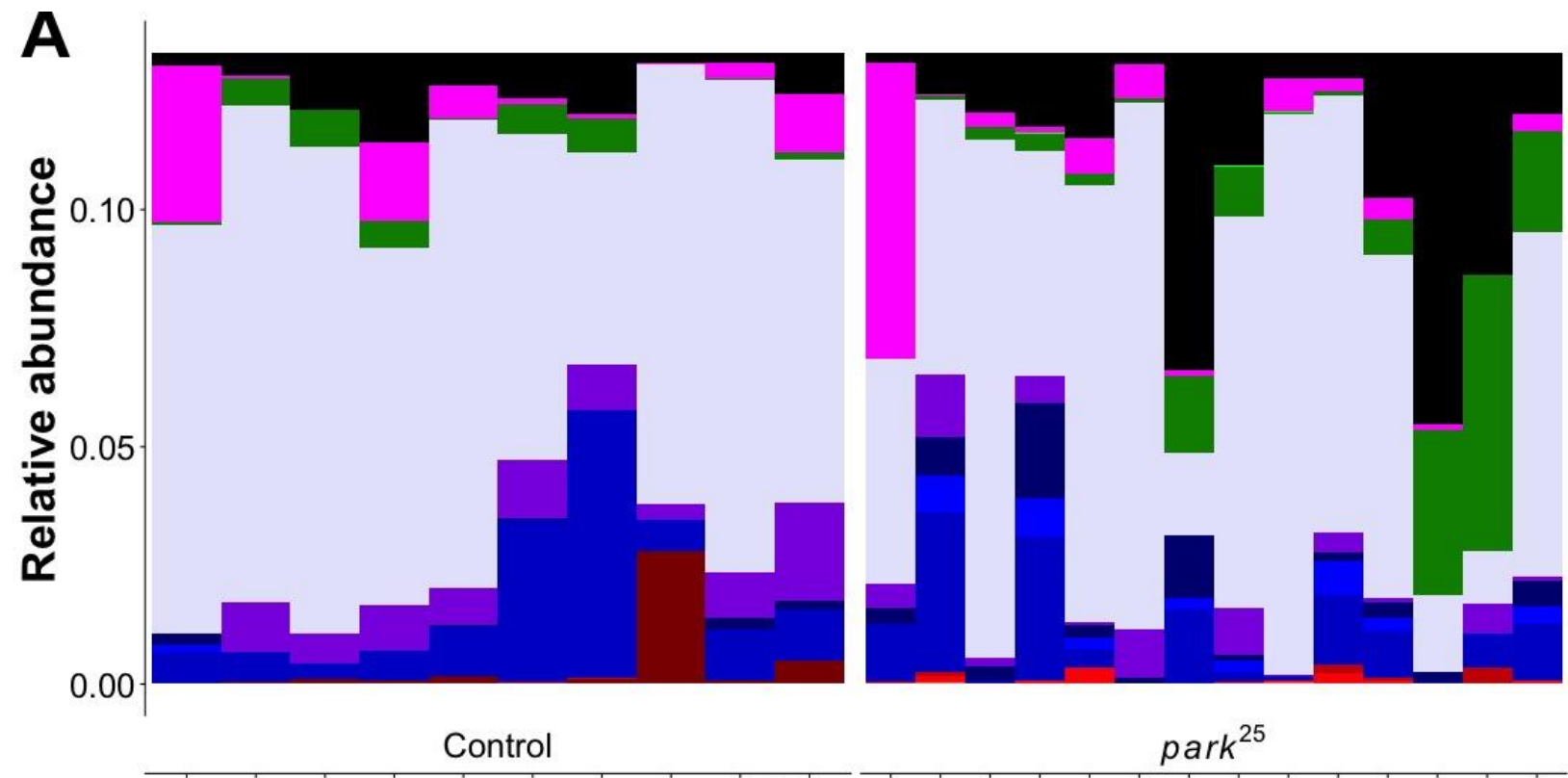

Samples

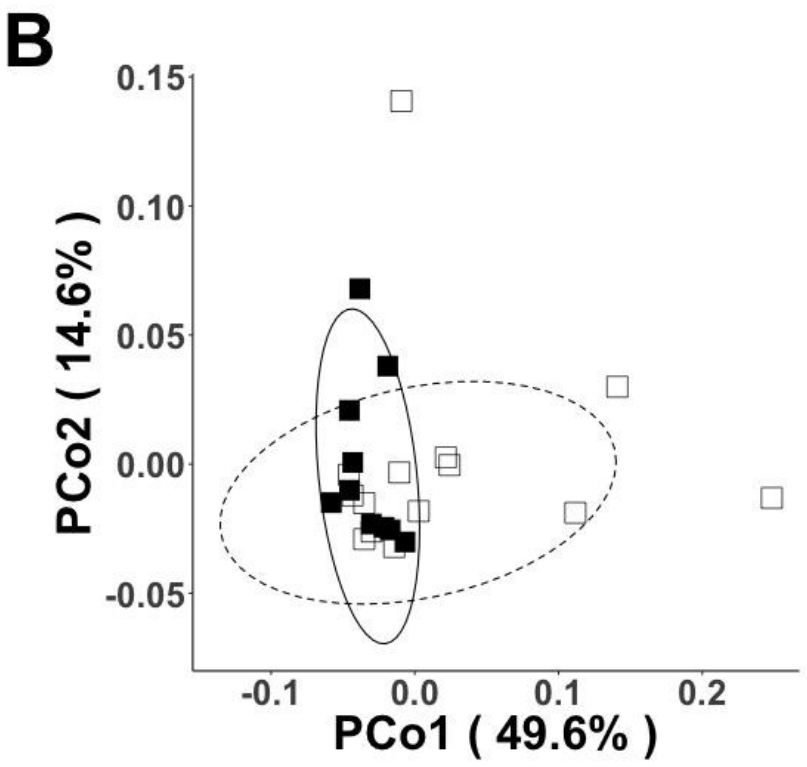

Other

C. variabile

Pasteurellales

Enterobacteriaceae

Enterococcus

Leuconostoc

L. brevis

Lactobacillus

Lactobacillaceae ASVs 1 and 2

Acetobacter ASV1

Acetobacter ASV2

Acetobacter ASV3

Acetobacter ASV4 
571 Figure 6. Mono-association with $A$. tropicalis or a combination of bacteria with park ${ }^{25}$ flies can alter

572 eclosion rate.

573 Embryos from park $^{25}$ flies were made axenic and then mono-associated with four different laboratory

574 bacterial strains, or inoculated with an equal CFU combination of the four strains (Combo). Pupae count

575 and eclosion was recorded from each vial. Heterozygous (Het) park ${ }^{25}$ pupae were differentiated from

576 the homozygous (Hom) park ${ }^{25}$ pupae by the presence of the Tubby marker on the TM6C balancer

577 chromosome. Data are presented as mean and SEM. Post-hoc Dunnett's analysis comparing to the

578 axenic control flies are shown: $* * * * \mathrm{P}<0.0001, * *=\mathrm{P}<0.01 *=\mathrm{P}<0.05$. Axenic $(n=160), \mathrm{L}$. brev $=$

579 Lactobacillus brevis $(n=24)$, L. plant = Lactiplantibacillus plantarum $(n=37)$, A. pom =

580 Acetobacter pomorum $(n=35)$, and A. trop = Acetobacter tropicalis $(n=37)$, combination $(n=32)$.

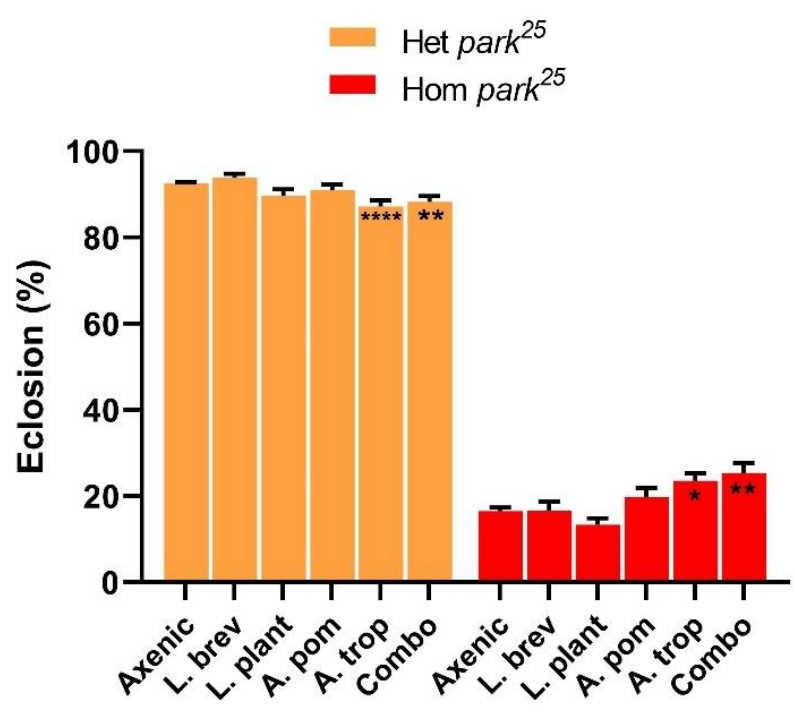

Bacterial status of experimental fly

581 


\section{Supplementary Files}

This is a list of supplementary files associated with this preprint. Click to download.

- MicrobiomeandDevelopmentSupplementarylnformation.pdf 